\title{
Complementary and Alternative Medicine Use in Cancer: The Role of Clinical Pharmacists
}

\author{
Amani E Khalifa* \\ Faculty of Pharmacy, Ain Shams University and Scientific Consultant for Children Cancer Hospital (CCHE 57357), Cairo, Egypt
}

*Corresponding author: Amani E Khalifa, Professor of Pharmacology \& Toxicology,

Faculty of Pharmacy, Ain Shams University, Cairo, Egypt.

Received Date: August 18, 2018

Published Date: August 27, 2018

Abbreviations: CAM: Complementary and Alternative Medicine; MCP: Modified Citrus Pectin; CAP: Complementary \& Alternative Products; CAPHP: Complementary \& Alternative Physical Health Practices; CAMHP: Complementary \& Alternative Mental Health Practices; T\&CM: Traditional \& Complementary Medicine

\section{Editorial}

Complementary and Alternative Medicine (CAM) refers generally to medical products and practices that are not part of standard medical care. Complementary medicine is the one that can be used together with conventional medicine. Alternative medicine is the one that can be used in place of conventional medicine. Integrative medicine refers to using conventional and complementary approaches together. CAM is becoming increasingly popular among the general population as well as among cancer patients. It is being used for the prevention and treatment of illness, holistic care, and/or counteracting the adverse effects of conventional medicine. Cancer patients in particular use CAM to cope with signs and symptoms caused by cancer and cancer medications such as anxiety, distress, uncertainty, confusion, fatigue, wasting, nausea \& vomiting, pain, difficulty sleeping, and stress. In one systematic review and meta-analysis, it has been reported that half of all cancer patients use CAM. The natural products were reported to be the most common complementary health approaches used by adults in different cultures and still another natural \& synthetic products are also being tested for potential beneficial effects against cancer or cancer-related side effects. For example, ginger root is being used either fresh, dried and powdered, or as a juice or oil in some cultures to treat nausea Cannabinoids may also be beneficial for nausea and vomiting associated with cancer. The effect of administering capsules of ground ginseng root have been studied in patients either during or after their treatment for cancer and patients who administered ginseng had less fatigue than patients who were given a placebo. L-carnitine; as a dietary supplement is also thought to be helpful in treating fatigue related to cancer. Coenzyme Q10; as an antioxidant compound made by the body to help cells produce energy, has been also reported to protect the heart from damage caused by certain chemotherapy drugs. Examples of some other antioxidants that may protect cells from the damage caused by free radicals that may lead to cancer are beta-carotene, lycopene, vitamins C, E, and A. It is to be noted that there has been some concern about whether antioxidants may make chemotherapy and radiation therapy less effective. Many studies suggest that some dietary supplements are commonly used by prostate cancer patients such as green tea, lycopene, modified citrus pectin, pomegranate, soy, and Zyflamend (a combination of a number of herbs) supplements. Modified Citrus Pectin (MCP) in particular have been reported to have effects on cancer growth and metastasis and it may protect against different types of cancer, including colon, lung, and prostate cancer. MCP is found in the peel and pulp of citrus fruits. There is also evidence that green tea may protect against different types of cancer since it contains polyphenol compounds including catechins, which are antioxidants that help protect cells from damage caused by free radicals. Oral glutamine; as an amino acid that can be synthesized by the body and can also be obtained from the diet, can reduce mucositis (often manifested as mouth sores) and diarrhea caused by chemotherapy and radiation therapy since it has been demonstrated to have a role in healing gastrointestinal mucosal cells and the entire GI tract. It has been claimed that antineoplastons; as drugs made of chemical compounds that occur naturally in the urine and blood, have selective effect in stopping growth of cancer cells without affecting healthy But, no randomized controlled trials showing 
the effectiveness of antineoplastons have been published in the peer-reviewed scientific literature. It has been also claimed that hydrazine sulfate; as a chemical compound, limits the ability of tumours to take in glucose that they need to grow. This compound has been studied as a treatment for cancer and as a treatment for cachexia/wasting that can develop with this disease. Nevertheless, hydrazine sulfate has shown no anticancer activity in randomized clinical trials, and data concerning its effectiveness in treating cancer-related cachexia are inconclusive [1-4].

In childhood cancer, the median estimated prevalence of $\mathrm{T} \& \mathrm{CM}$ use was reported to be $77 \%$ in low income countries (LIC), $67 \%$ in LMIC, $60 \%$ in UMIC, and $47 \%$ in HIC. The median rates of disclosure to healthcare providers though, differed significantly between LIC/ LMIC, UMIC, and HIC, with rates of $21 \%, 32 \%$, and $45 \%$, respectively. Nevertheless, CAM use can be harmful to cancer patients for example some CAM may indirectly induce the metabolism of cytotoxic agents, potentially leading to nontherapeutic systemic drug levels while other CAM may inhibit the metabolism of cytotoxic agents, leading to potentially lethal toxicities if the metabolic step inhibited inactivates the given drug. Moreover, there is a scarcity of published reports on specific CAM used and its impact on conventional cancer treatments. The association between its use and possible delays in presentation, diagnosis and treatments of cancer is also under investigated [5-6].

The NIH National Center for Complementary \& Integrative Health (NCCIH) is the US Government's lead agency for scientific research on complementary and integrative health approaches. NCCIH classified CAM into three categories; "Natural Products" as the first category, "Mind and Body Practices" as the second category, and "Other Complementary Health Approaches" as the third category. The first category of "Natural Products" is the most commonly used type of CAM among different populations and it includes herbs (botanicals), vitamins \& minerals, and probiotics. The second category of "Mind and Body Practices" includes practices such as yoga, chiropractic \& osteopathic manipulation, meditation, massage therapy, acupuncture, relaxation techniques (ex. breathing exercises, guided imagery, and progressive muscle relaxation), tai chi, qi gong, healing touch, hypnotherapy and movement therapies (such as Feldenkrais method, Alexander technique, Pilates, Rolfing Structural Integration, and Trager psychophysical integration). The third category of "Other Complementary Health Approaches" includes the practices of traditional healers, traditional Chinese medicine, Ayurvedic medicine (Indian), homeopathy and naturopathy. As an opinion, this classification could be argued against for the following reasons:

1-The first category of "Natural Products" is not comprehensive where it does not include all biological supplements having different sources of living organisms including animals, plants, fungi, and microorganisms (such as bacteria and archaea). In addition, any chemical complementary product being tested for potential therapeutic activity will not have a place under this category.

2- Acupuncture included in the second category is part of the traditional Chinese medicine included in the third category.
2- An overlap exists between natural products used as part of "naturopathy" approaches (that involves no surgery or synthetic drug use) included in the third category and the first category of "Natural Products".

4- The third category named "Other Complementary Health Approaches" combines mainly both products and practices which complicates things where they could be presented in a more simple way. For example, many products and practices used in Ayurvedic medicine such as herbs, massage, and specialized diets are also used on their own as complementary approaches. Similarly, traditional Chinese medicine practitioners also use herbs, acupuncture, and other practices, that can be used on their own as complementary approaches, to treat a wide range of conditions.

5- Naturopathy and Homeopathy listed in the third category is reported to lack an adequate/robust scientific basis, and it is rejected by a number of medical communities. Naturopathic physicians may claim to be primary care physicians proficient in the practice of both "conventional" and "natural" medicine. Their training, however, amounts to a small fraction of that of medical doctors who practice primary care. Another dilemma is that in the US naturopathy is also being practiced by traditional naturopaths who receive training that vary in length and content and are not accredited by organizations recognized for accreditation purposes by the U.S. Department of Education. In addition, neuropathy can be practiced by other health care providers such as physicians, osteopathic physicians, chiropractors, dentists, and nurses which adds to this dilemma. In 2015, the Australian Government's Department of Health published the results of a review of alternative therapies that sought to determine if any were suitable for being covered by health insurance; Naturopathy was one of 17 therapies evaluated for which no clear evidence of effectiveness was found. Homeopathy which promotes that disease can be cured by a substance that produces similar symptoms in healthy people and that the lower the dose of the medication, the greater its effectiveness, uses mainly "products" of plant, mineral, or animal-origin. In 2012, a research effort involving systematic review of case reports and case series concluded that using certain homeopathic products (such as those containing heavy metals like mercury or iron that are not highly diluted) or replacing an effective conventional treatment with an ineffective homeopathic one can cause adverse effects, some of which may be serious. A 2015 comprehensive assessment of evidence by the Australian government's National Health and Medical Research Council concluded that there is no reliable evidence that homeopathy is effective for any health condition. Finally, in December 2017, the U.S. Food and Drug Administration (FDA) proposed a new riskbased enforcement approach to homeopathic products [7-12].

Therefore, as an opinion, a more simple yet comprehensive classification of CAM could be suggested to include two categories; the first category of "Products" and the second category of "Practices". The first category will include both "Traditional (Culture-related)" and "Non-Traditional" products as subcategories. Similarly, the second category will include both "Traditional (Culture-related)" and "Non-Traditional" practices as 
subcategories. This "2Ps Classification" is valuable not only for its simplicity but also because it guides to a definite/clear/organized and hopefully universal responsibilities of three CAM practitioners among healthcare team in any hospital/clinical setting; Clinical pharmacists as the "Complementary \& Alternative Products' (CAP) Practitioner" responsible for the first "P" of CAM products [traditional (culture-related) and non-traditional] which is the most widely used type of CAM that also have potential interaction with conventional treatments. Physical therapists would be the "Complementary \& Alternative Physical Health Practices' (CAPHP) Practitioner" responsible for part of the second "P" of CAM practices [traditional (culture-related) and non-traditional]. Clinical Psychologists would be the "Complementary \& Alternative Mental Health Practices' (CAMHP) Practitioner" responsible for the rest of the second "P" of CAM practices [traditional (culturerelated) and non-traditional]. This "2Ps Classification" may also help to use universal terminology since many other terms that are sometimes inaccurate is being used for CAM in the literature such "Traditional \& Complementary Medicine" (T\&CM), "Natural Medicine", "Nonconventional Medicine", "Integrative Medicine", "Holistic Medicine", .

Knowing that success has been demonstrated in the treatment of HIV/AIDS in sub-Saharan Africa by integrating traditional health care practitioners in the healthcare team, it is important to stress the importance of investigating the role of clinical pharmacists in this dilemma. It is not advised that a new healthcare team members specialized in CAM be included among healthcare team members to add to this dilemma, but the pharmacists could be the one responsible for assuring the documentation of CAP information in the basic patient profile for the purpose of assisting clinicians \& policy makers to know specific CAP used and its impact on conventional treatments. Pharmacists can also help policy makers in formulating strategies and implement activities that can optimize the combined use of both complementary products and conventional therapies for many diseases in the future. From an academic perspective, it is suggested that the curricula of all undergraduate pharmacy programs should include complementary \& alternative medications/products (traditional and non-traditional) and their known interactions with conventional treatments. Also, curricula of all undergraduate physical therapy programs should include complementary \& alternative physical health practices (traditional and non-traditional). And the curricula of all undergraduate psychology programs should include complementary \& alternative mental health practices (traditional and non-traditional). Future research should be directed to investigating possible interactions of CAM with cancer conventional treatments, the extent to which CAM could contribute to the delay in the presentation, diagnosis and treatments, and the association between CAM use and adherence to cancer treatment and the optimization of combined use of both complementary and conventional therapies for the best interest of the patient.

\section{References}

1. Horneber M, Bueschel G, Dennert G, Less D, Ritter E, et_al. (2012) How Many Cancer Patients Use Complementary and Alternative Medicine: A Systematic Review and Metaanalysis. Integr Cancer Ther.11(3): 187203.

2. Cassileth BR, Deng G (2004) Complementary and Alternative Therapies for Cancer. Oncologist 9(1): 80-89.

3. Zulkipli AF, Islam T, Taib NAM, Dahlui M, Bhoo-Pathy N, et_al. (2017) Use of Complementary and Alternative Medicine Among Newly Diagnosed Breast Cancer Patients in Malaysia: An Early Report From the MyBCC Study. Integr Cancer Ther. 17(2):312-321

4. https://www.cancer.gov/about-cancer/treatment/cam/patient/camtopics-pdq\#link/_13_toc

5. Diorio C, Lam CG, Ladas EJ, Njuguna F, Afungchwi GM, et_al. (2017) Global Use of Traditional and Complementary Medicine in Childhood Cancer: A Systematic Review. J Glob Oncol 3(6): 791-800.

6. Tascilar M, de Jong FA, Verweij J, Mathijssen RHJ (2006) Complementary and Alternative Medicine During Cancer Treatment: Beyond Innocence. Oncologist. 11(7): 732-741.

7. https://nccih.nih.gov/health/integrative-health\#types

8. Jagtenberg T, Evans S, Grant A, Howden I, et al. (2006) Evidence-based medicine and naturopathy. J Altern Complement Med. 12(3): 323-328.

9. Atwood, Kimball C., IV. (2003) Naturopathy: A critical appraisal. MedGenMed 5(4): 39.

10. https://nccih.nih.gov/health/naturopathy

11. Baggoley C (2015) Review of the Australian Government Rebate on Natural Therapies for Private Health Insurance PDF. Australian Government - Department of Health. Lay summary - Gavura, S. Australian review finds no benefit to 17 natural therapies. ScienceBased Medicine.

12. https://nccih.nih.gov/health/homeopathy 